\title{
INVESTIGATION OF BEAM FORMING EFFECTIVENESS IN IEEE802.11AC INDOOR WIRELESS LINKS
}

\author{
Femi-Jemilohun Oladunni .Juliet, Walker Stuart. \\ School Of Computer Science and Electronic Engineering, University of Essex, \\ Colchester, Essex, United Kingdom \\ \{ojfemi and stuwal\} dessex.ac.uk
}

\begin{abstract}
The strong demand for large capacity transmission due to an unprecedented growth in data traffic requires an enhancement in the quality of service in the medium access control. This quality delivery features strongly in the IEEE802.11ac standard. Here, we have performed empirical measurements of an 802.11ac system based on the beam forming technology. In this work, an empirical analysis was conducted to determine the effectiveness of Beamforming Technology as depicts in the quality of service $(Q o S)$ enhancement in the newest wireless technology deployed on IEEE802.11ac standard. The experimental outcomes revealed the quality of service $(Q o S)$ enhancement in IEEE802.11ac. Although the realized throughput in real application scenario fell below designed specification. Nevertheless there is a considerable data throughput improvement on the counterpart 802.11n. There remain opportunities for further enhancements in beam-forming technology.
\end{abstract}

\section{KEYWORDS}

Beamforming, IEEE802.11ac, Shielding effect

\section{INTRODUCTION}

High data and file transfer rate through wireless service provision to an unlimited number of consumers is desired within the current limited frequency spectrum allocations The waiving of license in the microwave and millimetre wave frequency bands have attracted a lot of applications due to their ubiquitous and huge chunk of free spectrum. This has resulted in overcrowding in the microwave band, hence the experience in wireless communication has been that of poor quality of service, system degradation, as well as co-channel interference among the co-existing access networks $[1,2]$. Co-channel interference is a challenging problem in WLAN (wireless local area network) as well as WPAN (wireless personal area network. This has served as a major obstruction to reliable communication in these short-range wireless network systems. [3, 4]. An amendment to IEEE802.11 PHY and MAC layers has been studied by the IEEE802.11ac Task Group since 2008; to cope with the growing demands for wireless applications with higher data rate capability. IEEE802.11ac aims to improve upon its counterpart, IEEE802.11n. The framework for IEEE802.11ac stipulates support for an efficient channelization in wireless communication spectrum, hence improvement in spectrum utilization. Nevertheless, some unresolved technical challenges are inherent in this technology [5]. The emergence of this 
standard aims to give support to new applications such as uncompressed video streaming and sync-and-go at speeds above 1Gbps. Others include video demos/ tele-presence in auditoria, remote medical assistance, wireless networking for offices, medical imaging and surgical procedure support, in room gaming, broadcast TV field pick up, Desktop storage and display, and so on. [6] IEEE802.11ac defines use of eight spatial streams in spatial division multiplexing. Since this will be deployed in the less crowded and interference-free carrier frequency, it is hoped that the target of high throughput and enhancement in the entire wireless network capability and quality of service will be achieved.

While the initial IEEE 802.11 standard has little QoS support, a set of QoS enhancements to the Medium Access Control (MAC) forms the main part of IEEE 802.11ac, which is currently being specified. [6].

This work looks at the effectiveness of beam forming technology as a shielding technique to enhance the signal of interest and suppress interfering signals, thereby improving the performance of the upcoming technology deployed on IEEE802.11ac. This is achieved using the empirical and simulation based analyses. The results of the experiments are hereby presented with suggestion for future work for system capacity improvement. The rest of this paper is organized as follows: part two of section one is the review of past related works. The background study of related topics to this work was considered in section two. Experimental set ups and results were presented in section three. While section four showed the matlab simulation of IEEE802.11ac beamforming effectiveness, and the conclusion was presented in section five.

\subsection{Related Work}

A lot of shielding techniques for interference mitigation, improved system performances, and capacity through quality of service have been proposed by different authors. In [7], a PrimarySecondary transmission scheme was used to mitigate interference by beam forming and increase system capacity in WLAN systems. Matlab simulation was used [8] to investigate the efficiency of multi-cast beam forming optimization over IEEE802.11n WLAN.[9] proposed the combination of advanced distributed beam forming techniques at physical layer for the improvement of the overall network capacity. Analytical modelling to evaluate the performance of ad hoc networks with M-element array was conducted by [10].The upper and lower bounds on the transmission capacity in the multiple antenna ad hoc networks with multi-stream transmission and interference cancellation at the receiver were derived by [11] to increase system capacity through beam forming technique. [12] gave an overview of the newest technologies promised to deliver multigigabits throughput: IEEE802.11ac ad. They described the channelization PHY design, and MAC modifications and beam forming in the standards. [13] Developed an adaptive Beamforming algorithm capable of interference rejection in the Global Navigation Satellite System (GNSS) receivers. This will allow interference suppression using orthogonal projection to create nulls in the array pattern as well as enhancing the quality of signal [14], demonstrated how beam forming based on the MBER criterion can be used for the enhancement in performance gain in wireless communications. Using five sources and two-element antenna array, the BER of the MBER solution was compared with that of MMSE solution under three different conditions. It was concluded that the MBER beam former utilizes system resources more intelligently than the standard MMSE beamformer. [15] used a beamforming approach and a modified SalehValenzuela channel model with ray-tracing to mitigate interference in $60 \mathrm{GHz}$ wireless LAN. [16] Designed an advanced interference resilient scheme for asynchronous slow frequency hopping wireless personal area networking and time division multiple access cellular system for interference reduction in wireless system. Similar to [8] who used Matlab simulation to investigate the efficiency of multi-cast beamforming optimization over IEEE802.11n, [12] give an overview of the IEEE802.11ad standard in relation to gigabit throughput capacity through beam forming technology, its challenges, and techniques, this work is looking at the 
effectiveness of beam forming in the IEEE801.11ac using the empirical and Matlab simulation based analyses.

\section{BACKGROUND STUDY}

IEEE802.11 is a set of technological standards used in WLAN (wireless local area network) communications systems. They are operated in the $2.4,3.6$, and $5 \mathrm{GHz}$ frequency bands. The first in this group is IEEE80.11-2007 which has been subsequently amended as the need for higher capacity throughput is needed by the end users..[17-19] More importantly, the waiving of licenses in these bands has led to overcrowding, thus, the degradation of the network system performance by co-channel interference of the co-located networks. [7, 20]. The newest of these standards is the IEEE802.11ac, which promises to deliver upwards of 1 Gigabits throughput. IEEE802.11ac uses $80 \mathrm{MHz}$ channels to achieve $433 \mathrm{Mbps}$ in a single stream while its lower counterpart. IEEE $802.11 \mathrm{n}$ uses $40 \mathrm{MHz}$ channels to realize $150 \mathrm{Mbps}$ per stream. There is a maximum of $600 \mathrm{Mbps}$ realizable in the four streams of IEEE802.11n but IEEE802.11ac suggests a maximum of $6.93 \mathrm{Gbps}$ from its eight streams. A number of quality enhancement techniques have been incorporated into this standard for its better performance over the lower counterparts. Some of these are the MIMO, OFDM, higher-order modulation technique, and Beam forming technology targeted at improving the data transmission reliability. This standard will be deployed on the less congested band of 5GHz. [21-23]. TGac and TGad were established in Sep 2008 and Dec 2008 respectively, targeting completion of standardization late in 2012. [24-26]. The effect of beam forming technology in this standard is looked into in this paper..

\subsection{Antenna Beamforming Pattern}

This is a technique by which the gain pattern of an adaptive array is steered to a de-sired direction through beam or null steering process algorithms. Digital beam forming involves using digital signal processors to implement the algorithms. The Beamformer creates nulls towards the interference sources and simultaneously directs its mainlobe in the direction of the signal of interest for enhancement, thereby maintaining a gain in that direction. This enables the antenna system to direct the maximum antenna gain pattern to the desired users and steer nulls towards the interfering signals thereby reducing the effect of noise, co-channel interference, and other effects from undesired users which will degrade the signal quality. This causes improvement in overall performance of the system [27]. A sample antenna array pattern is shown in figure 1a below.

\subsection{Adaptive Beamforming Technology}

This is one of the latest technologies in wireless communication system. It uses Smart Antenna techniques to produce multiple beams concentration to enhance signal of interest, and at the same time places nullity on the direction of interfering signal. The Beamformer varies a weight vector for this adaptive process with the ultimate goal to improve communication channel. The figure $1 \mathrm{~b}$ is the setup model for adaptive beamforming. The mathematical algorithms are shown in the following equations [13]

$$
0<\mu<2 \frac{E \|\left. u(n)\right|^{2} \mid}{\left.E \|\left.\varepsilon(n)\right|^{2}\right]} D(n) \text {, Where }
$$

$E\left\lfloor|e(n)|^{2}\right\rfloor$ is the power of the difference between the output and reference signals $\mu$-step size $E\left\lfloor|u(n)|^{2}\right\rfloor$ is power of incoming signal and $D(n)$ is the deviation

The error signal is given by; 


$$
\begin{aligned}
& \mathrm{e}(n)=\mathrm{d}(n)-w^{{ }^{H}}(n) u(n) \\
& \hat{w}(n+1)={ }_{W}(n)+\frac{\mu}{\|u(n)\|^{2}} u(n) e^{*}(n) \\
& \mathrm{y}(n)=w^{H}(n) x(n), \\
& \mathrm{e}(n)=\mathrm{d}(n)-\mathrm{y}(n)
\end{aligned}
$$

Estimated of weight vector at time $n+1$,

$$
\begin{aligned}
& w(n+1)=w(n)+\mu x(n) e^{*}(n) \\
& =w(n)+\frac{\mu}{\|x(n)\|^{2}} x(n) e^{*}(n) \\
& =w(n)+\frac{\mu}{\|x(n)\|^{2}} x(n)\left\|d(n)-{ }_{w}^{H} x(n)\right\|^{*}
\end{aligned}
$$

The signal $\mathrm{x}(\mathrm{n})$ from multiple antenna elements is multiplied by a weight vector to adjust the phase and amplitude to give $\mathrm{y}(\mathrm{x})$. Least Mean Square algorithm is applied to minimise error e(n) between a set desired signal $d(n)$ and $y(n)$. Weight vectors are carefully chosen for the antenna array elements. These will allow the beamformer to adaptively steer the beam response to achieve maximum gain in the direction of signal of interest and nulls towards the interferers

\section{EXPERIMENTAL SETUP AND RESULTS}

To assess the performance of 802.11ac, an ad-hoc system with the latest technology in wireless standards was used. The AirStation 1750 (Buffalo) is amongst the first routers to implement the newest WiFi standard: IEEE802.11ac. It promises throughput of 1300Mbps and also additional $450 \mathrm{Mbps}$ in the 802.11n radio.[28] The setup for the experiments are shown in figs $1 \& 2$ using WLI-4H-D1300 and WZR-D 1800H for the transmission using 5GHz channels, For comparison, in fig two, the client was changed to a WNDA 3100 N600 (Net gear) dual-band dongle using the $5 \mathrm{GHz}$ channels of $802.11 \mathrm{n}$ The measurement of throughputs over a range of distances up to $30 \mathrm{~m}$ was observed on the PC connected to the wireless transmission link. The PCs were equipped with LANSpeedTest software for the monitoring of the throughputs.

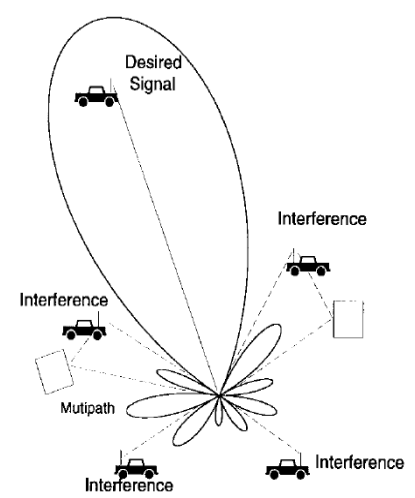

(a)

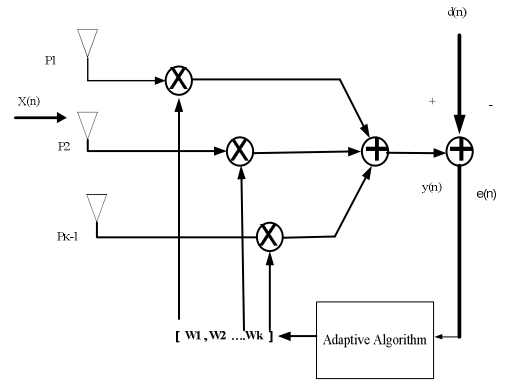

(b)

Fig. 1. (a) Antenna Array Beamforming [28], (b) Block Diagram of Adaptive Beamforming 

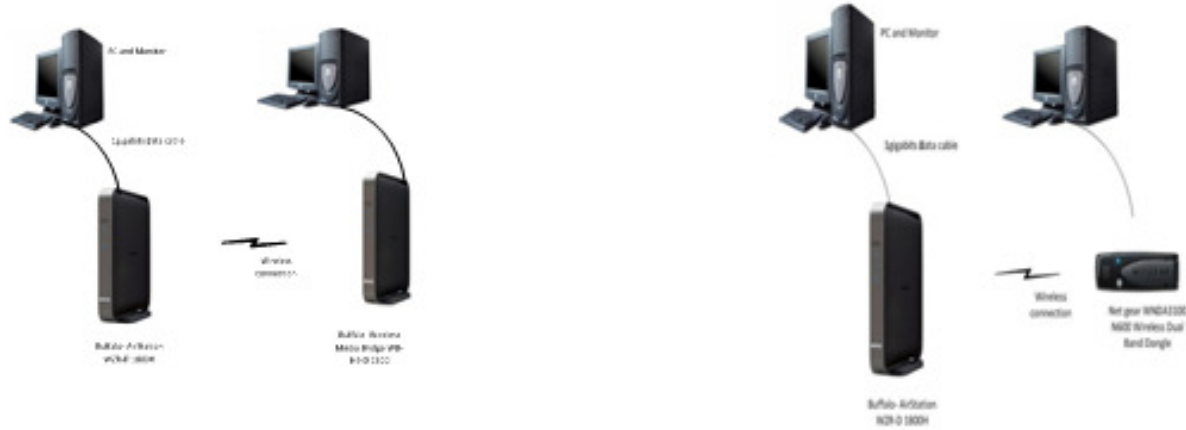

Figure. 2. Buffalo AirStation AC1300/N900 Gigabit Dual Band Wireless Router and Client. (a) With IEEE802.11ac Standard, (b) with Net gear WNDA3100N600 Wireless Dongle Receiver on IEEE802.11n.

\subsection{Process Adopted to Measure Throughput}

The setup shown in figs $2 \mathrm{a}$ and $2 \mathrm{~b}$ were used. The router was fixed in a position while the client/mobile terminal was moving at an equidistant points separated by $1 \mathrm{~m}$ until a total coverage of $30 \mathrm{~m}$ was reached in a laboratory hall where the measurement was conducted. The throughput levels received at each measure point is collected by a PC running with application software LANSpeed Test software, which was moving along various points of measurement to monitor the throughput from the client.

\subsection{Results of the Measurements}

Fig 3 shows the level of uplink and downlink throughputs of AC1300/N900 Gigabit Dual Band Wireless router and client when it is configured to work only in 802.11ac, and WNDA3100 N600 wireless dual-band dongle receiver when configured to work in $802.11 \mathrm{n}$. As the figure shows, the propagation square law was minimal and more revealed in IEEE802.11n configuration than IEEE802.11ac. The throughput in 802.11ac is higher than that of $802.11 \mathrm{n}$ Fig 4 shows the level of uplink and downlink throughputs of the AC1300/N900 Gigabit Dual Band Wireless router and client when it is configured to receive on $5 \mathrm{GHz}$, and WNDA3100 N600 wireless dual band dongle receiver when configured to receive on $2.4 \mathrm{GHz}$ but in cross polarization. Here, the propagation square law is obvious in both standards in appreciable measure. The throughput in $802.11 \mathrm{n}$ tends to reduce while in 802.11ac it is higher than when it was in the uplink link.. While the propagation square law is more revealing in $802.11 \mathrm{n}$, both standards have increased their throughput compared to the uplink performances 

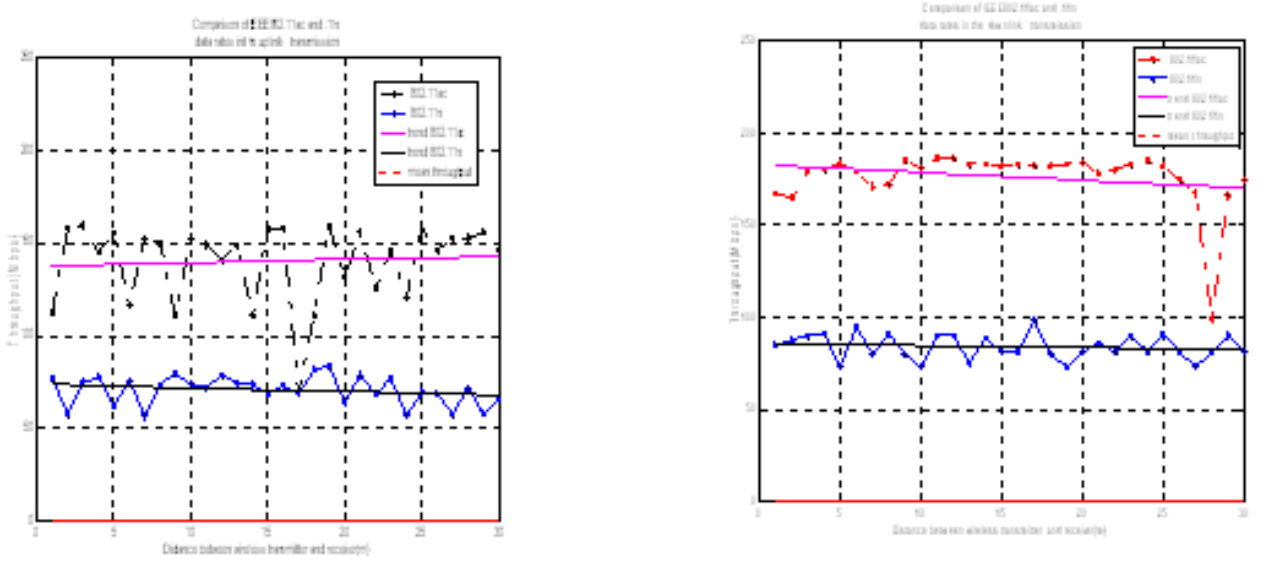

igure.3. Uplink and Downlink Transmission Throughputs with AirStation Receiver and Dongle Receiver
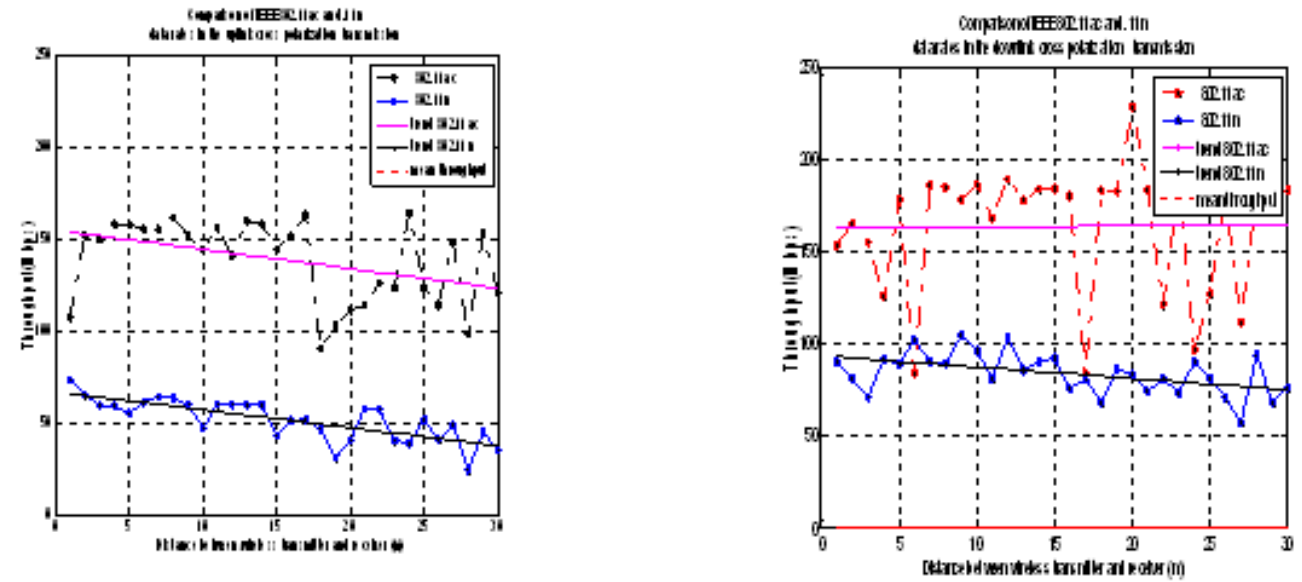

Figure 4. Uplink and Downlink Transmission Throughput with AirStation Receiver and Dongle Receiver in Cross polarization

\section{MATLAB SIMULATION OF BEAMFORMING IN IEEE802.11AC}

Table 1. Parameters for Simulation

\begin{tabular}{|l|l|}
\hline Carrier frequency & $5 \mathrm{GHz}$ \\
\hline Sampling frequency & $2 \mathrm{FC}$ \\
\hline Desires transmitted angle & $\mathrm{Pi} / 4$ \\
\hline Interferer transmitted angle & $\mathrm{Pi} / 2$ \\
\hline Step size & 0.32 \\
\hline Number of element antenna array & 8 \\
\hline
\end{tabular}




\subsection{Simulation Procedure}

Both the input signal $\mathrm{x}(\mathrm{n})$ and weight vector are multiplied together to produce the output signal $y(n)$. This results in a change in the amplitude and phase of the incoming signal. An error e(n) exist which is the difference between the output and a chosen reference signals $d(n)$. The adaptive algorithm tries to minimize this error. The step size must not exceed its upper bound as shown in equation 1. A LMS algorithm with a step size of 0.32 was used. Figure 5a shows the frequency spectrum of the desired and interfering signals after fast Fourier transform has been used with quantized raw signals to ease the application of weights at discrete level.

The higher magnitude in figure $5 \mathrm{~b}$ shows the desired signal and interfering signal before beamforming. The adaptive algorithm has adaptively suppressed the interfering signal after the beamforming as shown by the smaller magnitude representing the desired signal

\subsection{Observation}

There is appreciable measure of improved throughput in IEEE802.11ac over its counterpart IEEE802.11n. This established the benefits of beamforming technology in preserving the data to the targeted consumers. This is revealed in the graphs below. The magnitude of the desired and interfering signals is shown in red before beam-forming algorithm was implemented, while the blue colour signifies the magnitude of the desired signal after beamforming figure $5 \mathrm{~b}$. The drastic reduction in the magnitude is as a result of interference suppression through the shielding technique applied while the reception of desired signal is enhanced, hence improved quality of service.

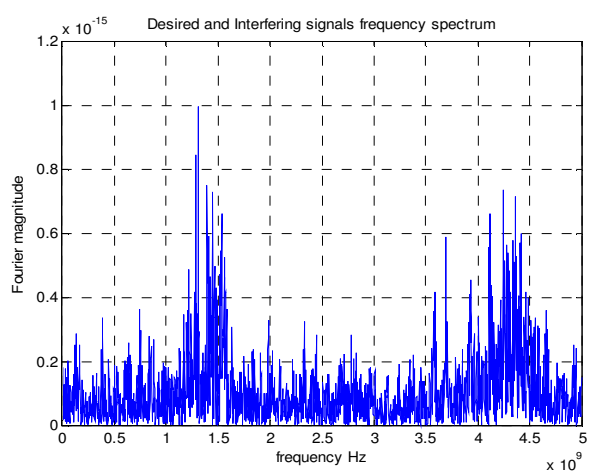

(a)

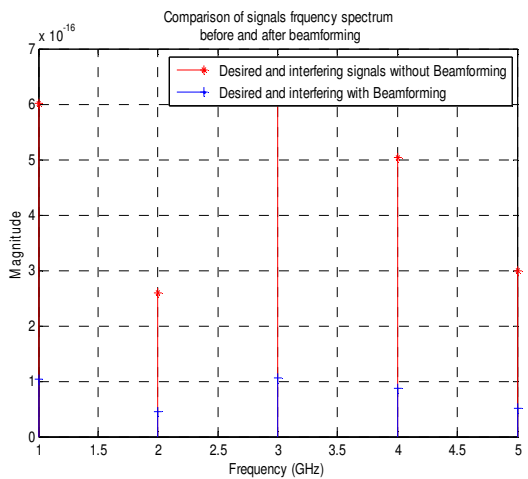

(b)

Fig. 5. ) Frequency Spectrum of Interfering and Desired Signals, (b) Frequency Spectrum before and after

\subsection{Limitation} Beamforming Technique

Electromagnetic fields as a result of the electronic devices in the Laboratory where this measurement was conducted are capable of causing variability through reflection and diffraction to the throughput.

Also significant variation could have been incurred as a result of people movements in close proximity to the transmitter or the receiver.

Due to the limited coverage area between the transmitter and receiver, variation in the direction of the antenna will result in corresponding high changes in the received throughput. Again, a lot of 
metallic objects in the laboratory, as well as materials like wood, crystal plastics and bricks could have reflected some of the signals, while objects with high humidity tendencies absorbed some signals. However, these are real situations where these wireless systems will be used.

\section{CONCLUSION}

The success in the growing wireless standards can be measured by the achievement of quality of service specifications by the designers. It becomes imperative to ascertain the performances of wireless devices[29]Though. the theoretical peak data rates of $600 \mathrm{Mbps}$ and $1.3 \mathrm{Gbps}$ promised in IEEE802.11ac and IEEE802.11n respectively.[30] may not be realizable due to propagation impairment contending with wireless transmission in the real world scenario, nevertheless there is a considerable data throughput improvement on the counterpart 802.11n. The experiments have shown that IEEE802.11ac outperforms its lower counterpart IEEE802.11n in throughput delivery. This is as a result of the beamforming technology incorporated into this standard as a shielding technique for enhancement of system performance. The beamforming technology could be conceivably be modified to have better performance. The simulation based evaluation depicted in figs 9 and 10 revealed the effectiveness of beamforming technique

\section{REFERENCES}

[1] K. K. Leung and B. J. Kim, "Frequency assignment for IEEE 802.11 wireless networks," in Vehicular Technology Conference, 2003. VTC 2003-Fall. 2003 IEEE 58th, 2003, pp. 1422-1426.

[2] H. Liu, H. Darabi, P. Banerjee, and J. Liu, "Survey of wireless indoor positioning techniques and systems," Systems, Man, and Cybernetics, Part C: Applications and Reviews, IEEE Transactions on, vol. 37, pp. 1067-1080, 2007.

[3] K. Kim, "Interference mitigation in wireless communications," 2005.

[4] A. Mahanti, N. Carlsson, C. Williamson, and M. Arlitt, "Ambient Interference Effects in Wi-Fi Networks," NETWORKING 2010, pp. 160-173, 2010.

[5] X. Wu, X. Zhang, Z. Wen, and Y. Wu, "A Solution for Chinese Efficient Channelization in IEEE 802.11 ac Wireless Network," 2011, pp. 1-4.

[6] I. Gutiérrez, G. Bacci, J. Bas, A. Bourdoux, M. Della Maggiora, H. Gierszal, A. Mourad, and S. Pfletschinger, "INFSCO-ICT-216203 DAVINCI D2. 1.4 v1. 0 Final Proposal for IMT Advanced systems."

[7] T. Murakami, R. Kudo, Y. Asai, T. Kumagai, and M. Mizoguchi, "Performance evaluation of distributed multi-cell beamforming for MU-MIMO systems," 2011, pp. 547-551.

[8] C. Papathanasiou and L. Tassiulas, "Multicast Transmission over IEEE $802.11 \mathrm{n}$ WLAN," in Communications, 2008. ICC'08. IEEE International Conference on, 2008, pp. 4943-4947.

[9] Y. Lebrun, K. Zhao, S. Pollin, A. Bourdoux, F. Horlin, S. Du, and R. Lauwereins, "Beamforming techniques for enabling spatial-reuse in MCCA 802.11 s networks," EURASIP Journal on Wireless Communications and Networking, vol. 2011, pp. 1-13, 2011.

[10] K. Fakih, J. F. Diouris, and G. Andrieux, "Analytical evaluation on the performance of ad hoc networks when using beamforming techniques," in Communications, 2008. ICC'08. IEEE International Conference on, 2008, pp. 2337-2342.

[11] R. Vaze and R. W. Heath, "Transmission capacity of ad-hoc networks with multiple antennas using transmit stream adaptation and interference cancellation," Information Theory, IEEE Transactions on, vol. 58, pp. 780-792, 2012.

[12] P. Xia, X. Qin, H. Niu, H. Singh, H. Shao, J. Oh, C. Y. Kweon, S. S. Kim, S. K. Yong, and C. Ngo, "Short range gigabit wireless communications systems: potentials, challenges and techniques," 2007, pp. 123-128.

[13] K. M. alias Jeyanthi and A. Kabilan, "A Simple Adaptive Beamforming Algorithm with interference suppression," International Journal of Engineering and Technology, vol. 1, pp. 1793-8236, 2009.

[14] S. Chen, N. Ahmad, and L. Hanzo, "Smart beamforming for wireless communications: a novel minimum bit error rate approach," 2002.

[15] M. Flament and A. Svensson, "Interference Mitigation in $60 \mathrm{GHz}$ Wireless Local Area Networks," 2007. 
[16] S. M. Alamouti, "A simple transmit diversity technique for wireless communications," Selected Areas in Communications, IEEE Journal on, vol. 16, pp. 1451-1458, 1998.

[17] A. Sandeep, Y. Shreyas, S. Seth, R. Agarwal, and G. Sadashivappa, "Wireless Network Visualization and Indoor Empirical Propagation Model for a Campus WI-FI Network," World Academy of Science, Engineering and Technology, vol. 42, 2008.

[18] G. Ofori-Dwumfuo and S. Salakpi, "WiFi and WiMAX Deployment at the Ghana Ministry of Food and Agriculture," Research Journal of Applied Sciences, vol. 3, 2011.

[19] J. J. van Rensburg and B. Irwin, "Wireless Network Visualization Using Radio Propagation Modelling," 2005.

[20] A. Mourad and A. Bourdoux, "INFSO-ICT-216203 DAVINCI D7. 2.2 v1. 0 Standardization and Regulatory Plan, Issue 2."

[21] M. Matsuo, M. Kurosaki, Y. Nagao, B. Sai, Y. Kuroki, A. Miyazaki, and H. Ochi, "HDTV over MIMO wireless transmission system," 2011, pp. 701-702.

[22] M. Matsuo, R. Ito, M. Kurosaki, B. Sai, Y. Kuroki, A. Miyazaki, and H. Ochi, "Wireless transmission of JPEG 2000 compressed video," 2011, pp. 1020-1024.

[23] E. Perahia and M. X. Gong, "Gigabit wireless LANs: an overview of IEEE 802.11 ac and 802.11 ad," ACM SIGMOBILE Mobile Computing and Communications Review, vol. 15, pp. 23-33, 2011.

[24] K. Nishikawa, "Ultra-High-speed Radio Communication Systems and Their Applications-Current Status and Challenges."

[25] R. Liao, B. Bellalta, and M. Oliver, "DCF/USDMA: Enhanced DCF for Uplink SDMA Transmissions in WLANs."

[26] M. Park, "IEEE 802.11 ac: Dynamic Bandwidth Channel Access," 2011, pp. 1-5.

[27] F. Rashid-Farrokhi, L. Tassiulas, and K. J. R. Liu, "Joint optimal power control and beamforming in wireless networks using antenna arrays," Communications, IEEE Transactions on, vol. 46, pp. 13131324, 1998.

[28] K. taylor. (2012, Buffalo AirStation 1750 802.11ac router review. IT ProPortal 24/7 Tech Commentary and Analysis.

[29] D. Sylvia, B. J. Mohan, and D. S. Rao, "Performance Evaluation of IEEE 802.11 b/n in Wireless Networks for QoS Improvement," International Journal, vol. 4, 2012.

[30] Q. Incorporated. (2012, IEEE802.11ac: The Next Evolution of Wi-Fi Standards. 15.

Femi-Jemilohun .O. Juliet is a Ph.D. candidate at the School of Computer Science and Electronic Engineering the University of Essex, Colchester, United Kingdom .She became a student member of IEEE in 2013.She received her B.Eng. degree in Electrical and Electronics engineering, Ondo State University, Ado Ekiti, Nigeria in 1997 and her MEng degree from Federal University of Technology, Akure, Ondo State, Nigeria in 2010.

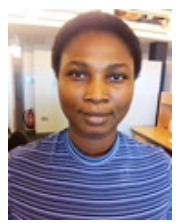

Stuart D. Walker received the B.Sc. (Hons.) degree in physics from Manchester University, Manchester, U.K., in 1973 and the M.Sc. and Ph.D. degrees in electronic systems engineering from the University of Essex, Essex, U.K., in 1975and 1981, respectively. After completing a period of contractual work forBritish Telecom Laboratories between 1979 and 1982, he joined the company as a Staff Member in 1982. He worked on various aspects of optical system design and wa spromoted to Head of the Regenerator

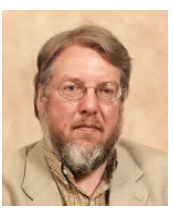
Design Group in 1987. In 1988, he became Senior Lecturer and then Reader in Optical Communication at the University of Essex, U.K. Currently, He is the Director of Postgraduate School,University of Essex. 